\title{
Pediatric screening tools for malnutrition: an update
}

\author{
Jessie M. Hulst ${ }^{\mathrm{a}}$, Koen Huysentruyt ${ }^{\mathrm{a}, \mathrm{b}}$, and Koen F. Joosten ${ }^{\mathrm{c}}$
}

\begin{abstract}
Purpose of review
There is ongoing interest in nutritional screening tools in pediatrics to facilitate the identification of children at risk for malnutrition who need further assessment and possible nutritional intervention. The choice for a specific tool depends on various factors. This review aims to provide an overview of recent progress in pediatric nutritional screening methods.
\end{abstract}

\section{Recent findings}

We present recent studies about newly developed or adjusted tools, the applicability of nutritional screening tools in specific populations, and how to implement screening in the overall process of improving nutritional care in the pediatric hospital setting.

\begin{abstract}
Summary
Three new screening tools have been developed for use on admission to hospital: two for the mixed pediatric hospitalized population and one for infants. A simple weekly rescreening tool to identify hospital-acquired nutritional deterioration was developed for use in children with prolonged hospital stay. Different from most previous studies that only assessed the relationship between the nutritional risk score and anthropometric parameters of malnutrition, new studies in children with cancer, burns, and biliary atresia show significant associations between high nutritional risk and short-term outcome measures such as increased complication rate and weight loss. For implementation of a nutritional care process incorporating nutritional screening in daily practice, simplicity seems to be of great importance.
\end{abstract}

Keywords

child, malnutrition, nutritional assessment, nutritional screening, undernutrition

\section{INTRODUCTION}

There is ongoing interest in nutritional screening tools (NSTs) in pediatrics to facilitate the identification of children at risk for malnutrition who need further assessment and possible nutritional intervention. Several recent (systematic) reviews of various available NSTs in pediatric patients have been published including information about each instrument's practicality, validity, accuracy, and comparison of different tools [1-9]. With this narrative review, we aimed at updating the reader's knowledge on newly developed tools, studies describing adjustments to previously developed NSTs, and their applicability in specific groups. Moreover, we describe studies addressing the use of screening in the process of improving nutritional care in the hospital setting. We selected studies with full-English manuscript available.

\section{NEW TOOLS AND ADAPTATION OF EXISTING TOOLS IN THE GENERAL HOSPITALIZED PEDIATRIC POPULATION}

Over the past 10 years, most publications considered the three most cited NSTs used in the general population of children admitted to the hospital: Pediatric

\footnotetext{
aDepartment of Paediatrics, Division of Gastroenterology, Hepatology and Nutrition, The Hospital for Sick Children, Toronto, Canada, 'Department of Pediatrics, Paediatric Gastroenterology, Universitair Ziekenhuis Brussel, Brussels, Belgium and 'Department of Pediatrics, Pediatric Intensive Care, Erasmus MC- Sophia Children's Hospital, Rotterdam, the Netherlands

Correspondence to Jessie M. Hulst, The Hospital for Sick Children, Division of Gastroenterology, Hepatology and Nutrition, Room 8260, 555 University Avenue, Toronto, ON, Canada M5G 1X8. ext 203656. Tel: +1 416 813-7654; e-mail: jessie.hulst@sickkids.ca
}

Curr Opin Clin Nutr Metab Care 2020, 23:203-209

DOI:10.1097/MC0.0000000000000644 


\section{KEY POINTS}

- Three new screening tools have been developed for use in a mixed pediatric population on admission to the hospital; one specific for the Chinese population [Pediatric Nutritional Screening Score (PNSS)], one focused on etiology-based risk factors [Pilot Pediatric Risk-Assessment Tool (PRAT)], and one for use in infants [Infant nutrition early warning score (iNews)].

- A simple, universal weekly rescreening tool to identify hospital-acquired nutritional deterioration [Pediatric Nutritional rescreening Tool (PNRT)] in children with a prolonged hospital stay was developed, which warrants further validation.

- Studies in children with specific diagnoses show significant associations between having a high nutritional risk score and short-term outcome measures such as increased length of stay, but also increase in complications and greater weight loss.

- Simplicity seems to be of great importance when implementing a nutritional care process in daily practice. Barriers for adequate nutrition support seem to be similar in all hospitals and are mainly issues around time, costs, and resources.

Yorkhill Malnutrition Score (PYMS), Screening Tool for the Assessment for Malnutrition in Pediatrics (STAMP), and Screening Tool for Risk of Impaired Nutritional Status and Growth (STRONGkids) [1012]. Although the published instruments have shown their clinical use, still new screening instruments are being developed or refined. Four recent studies will be discussed and their most important findings are summarized in Table 1.

In 2018, Lu et al. came up with the Pediatric Nutritional Screening Score (PNSS) specifically developed for the Chinese population. It was stated by the authors that STRONGkids, PYMS, and STAMP were developed by European researchers, but the selection and interpretation of these nutritional screening tools may differ among different racial and ethnic groups [13]. They also stated that it has been reported that the types and severity of diseases included in these screening tools are not sufficient to account for clinical diagnoses in China [14]. Therefore, they developed and validated the PNSS to assess undernutrition risk among hospitalized children in China. The development of the PNSS was based on the nutritional screening guidelines ESPEN [15] and modified according to Chinese clinical practice. PNSS consisted of three elements: disease with malnutrition risks (none or slight, moderate, severe); changes in food intake during the previous week; and nutritional status (assessed by anthropometric measurements). Each element received a score of $0-2$, with maximum total score of 6. Interestingly, the authors classified diseases into three different risk categories for malnutrition based on the presumed protein needs for the disease and the ability to be met with standard diet. From the 847 children included, $42.6 \%$ were at risk of undernutrition based on the PNSS results (cut-off score $\geq 2$ ). The score system of PNSS was calibrated following the assessment of body composition (using bio-impedance analysis). The sensitivity, specificity, and negative predictive value of PNSS using a complete dietetic assessment as reference method were 82,71 , and $92 \%$, respectively. Results were also compared with the three European screening tools. The sensitivity of PNSS was similar to that of STRONGkids, but higher than that of STAMP and PYMS.

Wong Vega et al. [16"'] stated that there is a lack of inclusion of cause-based risk factors in the

Table 1. Overview of new tools and adaptation of existing tools in the mixed pediatric hospitalized population

\begin{tabular}{|c|c|c|}
\hline Study & Screening tool & Considerations using screening tools \\
\hline Lu et al., 2018 [13] & PNSS & $\begin{array}{l}\text { Type and severity of disease with consequences for protein intake; } \\
\text { newly developed for Chinese population }\end{array}$ \\
\hline Wong Vega et al., 2019 [16""] & PRAT & $\begin{array}{l}\text { Focus on cause-based risk factors: wasting, hypermetabolism, } \\
\text { increased nutrient losses, altered absorption of nutrients, } \\
\text { inflammation }\end{array}$ \\
\hline White et al., 2019 [17"-] & PNRT & $\begin{array}{l}\text { Rescreening tool to be used weekly, starting } 7 \text { days after admission } \\
\text { (two questions): } \\
\text { Has the child had reduced nutritional intake in the last } 7 \text { days } \\
\text { Has the child lost weight or had poor weight gain }\end{array}$ \\
\hline Carter et al., 2019 [20] & $\begin{array}{l}\text { STRONGkids } \\
\text { and PNST }\end{array}$ & Use of different cut-offs to achieve a better risk classification \\
\hline
\end{tabular}

PNRT, Pediatric Nutritional Rescreening Tool; PNSS, Pediatric Nutritional Screening Score; PNST, Pediatric Nutrition Screening Tool; PRAT, Pilot Pediatric RiskAssessment Tool; STRONGkids, Screening Tool for Risk on Nutritional Status and Growth. 
previously published instruments and tested a Pilot Pediatric Risk Assessment Tool (PRAT). Therefore, a study in 528 hospitalized children was conducted to identify the prevalence of malnutrition and characterize mechanisms of malnutrition risk, and to relate these to outcome measures. Malnutrition was assessed by weight for length (WFL) and BMI/age Z-score, and overall prevalence was 19.7\%; 11.9\% mild, $5 \%$ moderate, and $2.8 \%$ severe. Antropometric z-scores were not associated with length of stay (LOS). Using the PRAT showed that altered absorption of nutrients and increased nutrient losses upon admission were independently associated with malnutrition on admission. Wasting, hypermetabolism, increased nutrient losses, and inflammation were associated with longer LOS. Interestingly, those with hypermetabolism had significant z-score improvements if followed by a dietician. The authors concluded that identification of risk factors beyond anthropometrics to define malnutrition and risk is important in prioritizing care.

White et al. designed and validated a simple, quick, and universal weekly rescreening tool to identify hospital acquired nutritional deterioration in 61 children with a prolonged LOS ( $\geq 7$ days) [17"']. Nutritional deterioration markers were collected by one investigator and two rescreening questions were asked by another investigator. Agreement between nutritional deterioration markers of reduction in weight $(\mathrm{kg})$, BMI $\left(\mathrm{kg} / \mathrm{m}^{2}\right)$, energy intake (kcal/day), and protein intake (g/day), and the two rescreening questions was determined. The sensitivity and specificity of the rescreening question 'Has the child had reduced nutritional intake in the last 7 days' for identifying children with an at least 25\% reduction in energy intake over the previous 7-day period were 61.9 and $82.2 \%$, respectively. The sensitivity of 'Has the child lost weight or had poor weight gain' $^{\prime}$ at detecting weight loss was $71.4 \%$ and specificity $87.8 \%$. The authors concluded that the pediatric nutrition rescreening questions provided a valid and simple tool to detect nutritional deterioration in long-stay pediatric patients and should be an integral part of the nutrition care process. The authors raised an important issue because no studies so far have been done that look at the validity of rescreening. The designers of the STRONGkids tool previously advised to repeat screening 1 week after admission, but no data of this rescreening have been published so far [18].

Carter et al. [20] wanted to determine which tool that can be easily used by nurses (STRONGkids [10] or PNST [19]) was able to identify children with malnutrition on hospital admission based on original and adjusted nutrition-risk cut-offs as compared to the subjective global nutritional assessment (SGNA). The reason to test STRONGkids and PNST was that these instruments consist of four 'yes-or-no' questions that can be completed in a few minutes and do not contain anthropometric measures. Compared to the 'golden standard' SGNA alternative cut-off points were derived, and using these alternative cut-off points, it was possible to achieve a better nutritional risk classification.

\section{USE OF NUTRITIONAL SCREENING TOOLS IN SPECIFIC PATIENT POPULATIONS}

Most studies on NSTs were performed in heterogeneous populations of hospitalized children with a variety of diseases [21]. Some studies have been performed in specific populations (disease, age, setting; see overview in Table 2); some of the new studies will be discussed in more detail below.

\section{Cancer}

In pediatric cancer, malnutrition is still a common complication and is related to outcome [22-25]. A recent single-center study [26"'] showed that performing a nutritional screening score had added value on top of anthropometric measurements only. Moreover, it is one of first studies to show a relationship between mortality, readmission rate, and nutritional status/risk. The study recruited 126 newly diagnosed cancer patients aged 3-18 years over a period of 5 years, and used STRONGkids to assess nutritional risk at diagnosis. The risk score was evaluated not only against nutritional status parameters (BMI z-score) but also importantly against outcome parameters such as survival and number of hospitalizations because of febrile neutropenia. At diagnosis, $28.6 \%$ of patients were at high risk of malnutrition, whereas $4.7 \%$ others were malnourished (BMI Z-score $\leq-2.0$ ). The risk of mortality and the rate of infections ( $\geq$ three hospitalizations for febrile neutropenia episodes) were significantly increased by malnutrition and rapid weight loss in the initial phase of treatment (3-6 months after diagnosis). The authors concluded that personalized evaluation of nutritional risk at diagnosis and close monitoring of nutritional status during the initial phase of treatment are crucial for ensuring appropriate intervention. This may potentially improve tolerance to chemotherapy and survival, and prevent prolonged hospitalization for infections in childhood cancer patients.

Another study in 70 pediatric patients (aged 1 month to 18 years) with recent cancer diagnosis [27] looked at the usefulness of a simplified and adapted version of the adult patient-generated 
Table 2. Overview of nutritional screening tools used in specific pediatric populations (diagnoses, settings, and age; ' $\mathbf{X}^{\prime}$ and names in bold font refer to the recent studies that came out in the review period; ' $x$ ' refers to previously published studies)

\begin{tabular}{|c|c|c|c|c|c|c|c|}
\hline & PNRS & STAMP & PYMS & STRONGkids & SGNA & iNews & Other \\
\hline \multicolumn{8}{|l|}{ Diagnoses } \\
\hline Anesthesia & $x[31]$ & & & & & & \\
\hline Biliary atresia & & & & $x[30]$ & & & \\
\hline Burns & & $x[29 "]$ & $x[29 "]$ & $x\left[29{ }^{\prime \prime}\right]$ & & & \\
\hline Cancer & & & & $x\left[26^{\prime \prime}\right]$ & x (PG-SGA) [27] & & SCAN tool [43] \\
\hline Cerebral palsy & & & & & $x[44]$ & & $\begin{array}{l}\text { Malnutrition Risk } \\
\text { Score [45] }\end{array}$ \\
\hline Cystic fibrosis & & & & & & & 2 NST $[46,47]$ \\
\hline IBD & & $x[48]$ & $x[48]$ & $x[48]$ & & & \\
\hline Spinal cord injury & & $x[49] / x[50]$ & & & & & \\
\hline Surgical patients & & & & $x[51]$ & & & \\
\hline \multicolumn{8}{|l|}{ Setting } \\
\hline $\begin{array}{l}\text { Chronic illness (mixed)- } \\
\text { special schools }\end{array}$ & & & & $x[52]$ & & & \\
\hline Ambulatory clinic & & $x[53]$ & & & & & \\
\hline \multicolumn{8}{|l|}{ Age } \\
\hline Infants & & $x[54]$ & & $x[36]$ & $x[35]$ & $x\left[33^{-"}\right]$ & NNST[55] \\
\hline
\end{tabular}

iNews, Infant Nutrition Early Warning Score; NNST, Neonatal Nutritional Risk Score; PG-SGA, Patient-generated Subjective Global Assessment; PNRS, Pediatric Nutritional Risk Screening; PYMS, Pediatric Yorkhill Malnutrition Score; SCAN, Nutrition Screening Tool for Childhood Cancer; SGNA, subjective global nutritional assessment; STAMP, Screening Tool for the Assessment for Malnutrition in Pediatrics; STRONGkids, Screening Tool for Risk on Nutritional Status and Growth.

subjective global assessment (PG-SGA) [28] to identify nutritional risk. The PG-SGA consisted of information about weight, height, food intake, gastrointestinal symptoms, functional capacity, physical exploration, and also the presence of ascites and edema. It divided the patients into four groups: well nourished $(78.6 \%)$, mildly $(17.1 \%)$, moderately $(4.3 \%)$, and severely (0\%) malnourished. The authors concluded that the PG-SGA is a valid tool because the correlations and the concordance between the PG-SGA, and anthropometric indicators were moderate and significant.

\section{Burns}

A prospective study [29"] assessed the nutritional risk of 100 children hospitalized with acute burn injuries and their associated clinical outcomes using STRONGkids, PYMS, and STAMP. The screening tools identified varying percentages of high-risk patients $(16,45$, and $46 \%$ by STRONGkids, PYMS, and STAMP, respectively). Interestingly, after adjusting for confounding factors, high-risk patients using either tool had significantly longer median LOS and greater median weight loss. Worse-than-average clinical outcomes were better predicted by the NRS tools than BMI z-score and burn severity.

\section{Biliary atresia}

In a study [30] among 106 patients diagnosed with biliary atresia type III, which aimed to explore the effect of preoperative nutritional status on cholangitis of a Kasai portoenterostomy, STRONGkids identified $46.2 \%$ of patients with moderate nutritional risky and 53.8\% patients with high risk preoperatively. The moderate-risk group had shorter postoperative LOS than the high-risk group, later initial occurrence of postoperative cholangitis and lower incidence of early cholangitis compared to the high-risk group. The ratio of jaundice clearance and 2-year native liver survival were significantly higher in moderate-risk group than in high-risk group.

\section{Patients undergoing general anesthesia}

A large French cross-sectional observational study including 985 patients ( $<18$ years) aimed to estimate the frequency of malnutrition and identify associated factors in children undergoing anesthesia [31]. Malnutrition rates were found to be 7.6, 8.1, and $11 \%$ when based on Waterlow index below $80 \%$, clinical signs, and when defined by a BMI less than P3, respectively. In multivariate analysis, a premature birth, a lower birth weight, and a 
higher Pediatric Nutritional Risk Score [32] were significantly associated with a higher odds of malnutrition when defined by BMI. No information was given on the outcome of the patients or on association between nutritional risk/status and outcome.

\section{Infants}

Because malnutrition during infancy can have longterm adverse consequences for both physical and psychological development, and most previously described screening tools exclude infants and children below 30 days of age (STRONGkids, PediSmart) or under 1 year (PYMS) or 2 years (STAMP) of age, there is interest in looking at infant age separately. Recent studies in infants have used three different nutritional risk tools, that is, newly developed Infant Nutrition Early Warning Score (iNews) [33"-], SGNA [34], and STRONGkids [10]. The iNews tool [33"'] was studied in 499 hospitalized infants in the United Kingdom, Greece, and Iran, and aimed to develop a tool to identify sick infants in need of dietetic input. Simple screening questions were used in the UK cohort to formulate iNEWS, which was then validated in the other groups. Based on dietetic assessment, 9.6 and $22 \%$ of UK and Greek infants, respectively, were rated as needing dietetic input. Being underweight, having poor weight gain/loss, and reduced intake were all independent predictors of perceived need for dietetic input. The iNEWS based on these items had 84\% sensitivity, 91\% specificity, and $49 \%$ positive predictive value to predict need for dietetic input in the UK cohort, which seems promising. In another study, 110 hospitalized infants were investigated using SGNA and z-scores based on anthropometric measurements [35]. Based on SGNA, 27.3\% were classified as moderately malnourished and $1.8 \%$ as severely malnourished, whereas acute (WFL z-score $<-2$ ) and chronic (LFA z-score $<-2$ ) malnutrition was found in 16.4 and $3.6 \%$, respectively. Lower z-scores were found in infants identified as malnourished using SGNA. Decrease in serial weight, having prolonged gastrointestinal symptoms, and reduced nutrition-related functional capacity were associated with malnutrition after adjusting for other factors.

Another cross-sectional study performed in 500 hospitalized children aged below 3 years in a children's hospital in Egypt found that $37.8 \%$ of patients were high risk according to STRONGkids, $62.4 \%$ were underweight, $58.4 \%$ were stunted, and $57.8 \%$ were wasted according to WHO criteria [36]. The STRONGkids score was found to be higher among those who deteriorated in nutritional status, which, together with its significant positive correlation with the duration of hospital stay, emphasized that STRONGkids score could be a predictive tool.

\section{SCREENING AND HOSPITAL NUTRITION CARE PROCESS}

Nutritional screening is considered a key part of the hospital nutrition care process and pediatric nutritional care algorithm [37]. A number of recent studies have focused on the organizational aspect of nutritional screening, focusing on auditing local or national practices $\left[38,39^{\mathbf{2}}, 40\right]$, describing barriers hindering optimal nutrition care $\left[3^{-}, 40,41\right]$, and propositions to simplify or improve current practices $\left[38,39^{\prime}, 42\right]$. In a 1-day cross-sectional audit, it was found that only $17 \%$ of the 113 admitted children had all phases ('diagnosis,' current intake, anthropometry) of their NST, which is an adapted version of the STAMP, completed. The authors identified the acquisition and plotting of growth parameters as one of the barriers (although compliance with height measurements were significantly improved compared to similar audits 5 years and a decade ago) and implemented automatically calculated z-scores into their electronic medical record system as a response to this. Furthermore, the use of a 'score' for nutritional screening was abandoned and replaced by clinical assessment in the hope of including the whole team into the screening process [38]. A Belgian study investigated their nutrition care process and more specifically their locally designed 'EvalNut' software program that combines the use of the PNRS screening tool with recommendations for nutritional management, including weekly follow-up [39"]. When reviewing the nutritional care data of 2657 pediatric inpatients, $35 \%$ of the evaluations were found to be incomplete or incorrect due to ambiguity of entrance data required for the software, missing/erroneous anthropometric recordings and too demanding nature of the amount of input needed. Simplification measures (reduction of the amount, internal validation and standardization of input needed, integration into the electronic medical health records, and automated alerts) were proposed to improve compliance.

A nationwide survey sent out to all members of the Korean Society of Pediatric Gastroenterology, Hepatology and Nutrition working in pediatric inpatient care revealed that only $52.5 \%$ of 40 participating hospitals had a nutrition support team [40]. Nutritional screening practices were less commonly performed for pediatric (55\% of the centers) than adult patients (65.8\% of the centers). Most pediatric centers used a locally developed screening tool, although two centers implemented a validated screening tool (PNRS 
and STRONGkids). The survey identified a lack of time and manpower, inexperience, low financial reimbursement for nutrition care, and unavailable resources as the main barriers for nutritional support [40]. The same main barriers were named in a multicenter international survey among 693 pediatric gastrointestinal doctors and dieticians: low staff awareness on nutrition, lack of time and lack of local guidelines for nutrition screening, but also the absence of local guidelines or policy to screen [41]. This survey also showed that overall only $23 \%$ of the responders used validated nutritional screening tools in their clinical practice, although depending on the country, this could go up to $50 \%$.

Most recently, the impact of the introduction of STAMP on nutritional status awareness among the medical staff was investigated by interpreting nutrition-related and anthropometric data from 182 medical records before and after implementing STAMP. No significant increase in acquisition of anthropometric data was found and moreover, virtually none of the patients were getting height measurements before or after the introduction of STAMP [42]. This shows that implementing a screening tool is not enough to acquire a mentality change in daily practice. It needs to be imbedded in a broader package of measures and training for the entire medical staff.

\section{CONCLUSION}

Over the past years, three NSTs used for children admitted to hospital were most frequently cited: PYMS, STAMP, and STRONGkids [10-12]. Although these published tools have shown their clinical use, these and other NSTs are studied in disease-specific populations, and are being refined or further developed for specific groups (infants) or populations (Chinese). All studies about previously and newly developed tools have shown their usefulness but also their limitation in clinical practice. There are numerous barriers hindering the routine use of NSTs, which hinders also appropriate nutritional support. Creating further awareness and simplification of the overall nutritional care process, which may include a NST, seems to be very important and the focus of future studies in order to improve nutritional care in hospitals.

\section{Acknowledgements}

None.

\section{Financial support and sponsorship}

None.

\section{Conflicts of interest}

There are no conflicts of interest.

\section{REFERENCES AND RECOMMENDED}

\section{READING}

Papers of particular interest, published within the annual period of review, have been highlighted as:

- of special interest

- of outstanding interest

1. Rinninella E, Ruggiero A, Maurizi $P$, et al. Clinical tools to assess nutritional risk and malnutrition in hospitalized children and adolescents. Eur Rev Med Pharmacol Sci 2017; 21:2690-2701.

2. Hulst JM, Joosten KFM. Nutrition screening: coding after discharge underestimates the prevalence of undernutrition. J Acad Nutr Diet 2018; 118:33-36.

3. Dos Santos $C A$, Ribeiro $A Q$, Rosa $C O B$, et al. Nutritional risk in pediatrics by StrongKids: a systematic review. Eur J Clin Nutr 2019; 73:1441-1449.

4. Aponte Borda AD, Pinzon Espitia OL, Aguilera Otalvaro PA. [Nutritional screening in hospitalized pediatric patients: systematic review]. Nutr Hosp 2018; 35:1221-1228.

5. Lee YJ. Nutritional screening tools among hospitalized children: from past and to present. Pediatr Gastroenterol Hepatol Nutr 2018; 21:79-85.

6. McCarthy A, Delvin E, Marcil V, et al. Prevalence of malnutrition in pediatric hospitals in developed and in-transition countries: the impact of hospital practices. Nutrients 2019;11:236.

7. Klanjsek P, Pajnkihar M, Marcun Varda N, et al. Screening and assessment tools for early detection of malnutrition in hospitalised children: a systematic review of validation studies. BMJ Open 2019; 9:e025444.

8. Marino LV, Thomas PC, Beattie RM. Screening tools for paediatric malnutrition: are we there yet? Curr Opin Clin Nutr Metab Care 2018; 21:184-194.

9. Delvin E, Harrington DJ, Levy E. Undernutrition in childhood: clinically based assessment tools and biological markers: where are we and where should we go? Clin Nutr ESPEN 2019; 33:1-4.

10. Hulst JM, Zwart $\mathrm{H}$, Hop WC, et al. Dutch national survey to test the STRONGkids nutritional risk screening tool in hospitalized children. Clin Nutr 2010; 29:106-111.

11. Gerasimidis K, Macleod I, Maclean A, et al. Performance of the novel Paediatric Yorkhill Malnutrition Score (PYMS) in hospital practice. Clin Nutr $2011 ; 30: 430-435$.

12. McCarthy HDM, Crabtree I, Eaton-Evans MJ, McNulty $H$. The development and evaluation of the Screening Tool for the Assessment of Malnutrition in Paediatrics (STAMP(c)) for use by healthcare staff. J Hum Nutr Diet 2012; 25:311-318.

13. Lu L, Mao X, Sheng J, et al. Development and validation of a pediatric nutritional screening score (PNSS) for hospitalized children. Asia Pac J Clin Nutr 2018; 27:65-71.

14. Cao J, Peng L, Li R, et al. Nutritional risk screening and its clinical significance in hospitalized children. Clin Nutr 2014; 33:432-436.

15. Kondrup J, Allison SP, Elia M, et al. ESPEN guidelines for nutrition screening. Clin Nutr 2003; 22:415-421.

16. Wong Vega $M$, Beer $S$, Juarez $M$, et al. Malnutrition risk in hospitalized children: a

-1 descriptive study of malnutrition-related characteristics and development of a pilot pediatric risk-assessment tool. Nutr Clin Pract 2019; 34:406-413.

New concept by the authors introduced looking at cause-based reasons for malnutrition and risk. Focus also on the dietician-based physical assessment to predict length of hospital stay and need for intervention.

17. White MS, Ziemann M, Doolan A, et al. A simple nutrition screening tool to identify

- nutritional deterioration in long stay paediatric inpatients: the Paediatric Nutrition Rescreening Tool (PNRT). Clin Nutr ESPEN 2019; 34:55-60.

First study investigating two rescreening questions 7 days after admission, which provide a simple and validated tool to detect nutritional deterioration.

18. Joosten KFM, Hulst JM. Nutritional screening tools for hospitalized children: methodological considerations. Clin Nutr 2014; 33:1-5.

19. White $M$, Lawson $K$, Ramsey $R$, et al. Simple nutrition screening tool for pediatric inpatients. JPEN J Parenter Enteral Nutr 2016; 40:392-398.

20. Carter LE, Shoyele G, Southon $S$, et al. Screening for pediatric malnutrition at hospital admission: which screening tool is best? Nutr Clin Pract 2019; doi: 10.1002/ncp.10367 [Epub ahead of print]

21. Huysentruyt $K$, Vandenplas $Y$. De Schepper J Screening and assessment tools for pediatric malnutrition. Curr Opin Clin Nutr Metab Care 2016; 19:336-340.

22. Loeffen EA, Brinksma A, Miedema KG, et al. Clinical implications of malnutrition in childhood cancer patients: infections and mortality. Support Care Cancer 2015; 23:143-150.

23. Pribnow AK, Ortiz R, Báez LF, et al. Effects of malnutrition on treatmentrelated morbidity and survival of children with cancer in Nicaragua. Pediatr Blood Cancer 2017; 64:e26590.

24. Norman $\mathrm{K}$, Pichard $\mathrm{C}$, Lochs $\mathrm{H}$, et al. Prognostic impact of disease-related malnutrition. Clin Nutr 2008; 27:5-15.

25. Rogers PC. Nutritional status as a prognostic indicator for pediatric malignancies. J Clin Oncol 2014; 32:1293-1294

26. Triarico $S$, Rinninella $E$, Cintoni $M$, et al. Impact of malnutrition on survival and

- infections among pediatric patients with cancer: a retrospective study. Eur Rev Med Pharmacol Sci 2019; 23:1165-1175.

This single-center study in pediatric oncology patients is one of first studies to show a relationship between mortality, readmission rate, and nutritional status/risk. 
27. Vazquez de la Torre MJ, Stein $K$, Vasquez Garibay EM, et al. PatientGenerated Subjective Global Assessment of nutritional status in pediatric patients with recent cancer diagnosis. Nutr Hosp 2017; 34:1050-1058.

28. Bauer J, Capra S, Ferguson M. Use of the scored Patient-Generated Subjective Global Assessment (PG-SGA) as a nutrition assessment tool in patients with cancer. Eur J Clin Nutr 2002; 56:779-785.

29. Bang YK, Park MK, Ju YS, et al. Clinical significance of nutritional risk

- screening tool for hospitalised children with acute burn injuries: a crosssectional study. J Hum Nutr Diet 2018; 31:370-378.

Interesting study showing that worse-than-average clinical outcomes were better predicted by the nutritional risk screening tools than by BMl z-score and burn severity.

30. Li D, Chen X, Fu K, et al. Preoperative nutritional status and its impact on cholangitis after Kasai portoenterostomy in biliary atresia patients. Pediatr Surg Int 2017; 33:901-906.

31. Gerbaud-Morlaes L, Frison E, Babre F, et al. Frequency and risk factors for malnutrition in children undergoing general anaesthesia in a French university hospital: a cross-sectional observational study. Eur J Anaesthesiol 2017; 34:544-549.

32. Sermet-Gaudelus I, Poisson-Salomon AS, Colomb V, et al. Simple pediatric nutritional risk score to identify children at risk of malnutrition. Am J Clin Nutr 2000; 72:64-70.

33. Gerasimidis $K$, Milani $S$, Tester $A$, et al. A multicentre development and

n. evaluation of a dietetic referral score for nutritional risk in sick infants. Clin Nutr 2018; 38:1636-1642.

Large well designed multicenter study to validate a dietetic referral score for nutritional risk in infants.

34. Secker DJ, Jeejeebhoy KN. Subjective global nutritional assessment for children. Am J Clin Nutr 2007; 85:1083-1089.

35. Matsuyama $M$, Bell $K$, White $M$, et al. Nutritional assessment and status of hospitalized infants. J Pediatr Gastroenterol Nutr 2017; 65:338-342.

36. Shaaban $\mathrm{S}$, Nassar M, El-Gendy $Y$, et al. Nutritional risk screening of hospitalized children aged $<3$ years. East Mediterr Health J 2019; 25:18-23.

37. Huysentruyt K, De Schepper J, Bontems P, et al. Proposal for an algorithm for screening for undernutrition in hospitalized children. J Pediatr Gastroenterol Nutr 2016; 63:e86-e91.

38. Marino LV, Beattie RM. Compliance with nutrition screening in a children's hospital. Arch Dis Child 2018; 103:798-800.

39. De Longueville $\mathrm{C}$, Robert $\mathrm{M}$, Debande $\mathrm{M}$, et al. Evaluation of nutritional care of

hospitalized children in a tertiary pediatric hospital. Clin Nutr ESPEN 2018; 25:157-162.

Large study describing the experience in introducing and refining a nutritional screening tool in daily clinical practice

40. Kim S, Lee EH, Yang HR. Current status of nutritional support for hospitalized children: a nationwide hospital-based survey in South Korea. Nutr Res Pract 2018; 12:215-221.
41. Marderfeld L, Rub G, Hodik G, et al. Validation and impact of paediatric malnutrition screening tool in hospitalised children on awareness of medical staff and health-related outcomes. Nutr Diet 2019; 76:574-579.

42. Huysentruyt $\mathrm{K}$, Hulst J, Bian $\mathrm{F}$, et al. Opinions and practices of healthcare professionals on assessment of disease associated malnutrition in children: results from an international survey. Clin Nutr 2019; 38:708-714.

43. Murphy AJ, White $M$, Viani $K$, et al. Evaluation of the nutrition screening tool for childhood cancer (SCAN). Clin Nutr 2016; 35:219-224.

44. Minocha $P$, Sitaraman $S$, Choudhary $A$, et al. Subjective global nutritional assessment: a reliable screening tool for nutritional assessment in cerebral palsy children. Indian J Pediatr 2018; 85:15-19.

45. Johnson A, Gambrah-Sampaney C, Khurana E, et al. Risk factors for malnutrition among children with cerebral palsy in Botswana. Pediatr Neurol 2017; 70:50-55.

46. Souza Dos Santos Simon MI, Forte GC, da Silva Pereira J, et al. Validation of a nutrition screening tool for pediatric patients with cystic fibrosis. J Acad Nutr Diet 2016; 116:813-818.

47. McDonald CM. Nutrition screening and diagnosis with early, appropriate interventions are critical components of cystic fibrosis management. J Acad Nutr Diet 2016; 116:771-773.

48. Wiskin AE, Owens DR, Cornelius VR, et al. Paediatric nutrition risk scores in clinical practice: children with inflammatory bowel disease. J Hum Nutr Diet 2012; 25:319-322.

49. Wang YJ, Zhou HJ, Liu PJ, et al. Risks of undernutrition and malnutrition in hospitalized pediatric patients with spinal cord injury. Spinal Cord 2017; 55:247-254.

50. Wong S, Graham A, Harini SP, et al. Profile and prevalence of malnutrition in children with spinal cord injuries-assessment of the Screening Tool for Assessment of Malnutrition in Paediatrics (STAMP). Spinal Cord 2012; 50:67-71.

51. Durakbaşa ÇUFS, Bayar $A$, Mutus $M$, Okur $H$. The prevalence of malnutrition and effectiveness of STRONGkids tool in the identification of malnutrition risks among pediatric surgical patients. Balkan Med J 2014; $31: 313-321$.

52. Joosten $\mathrm{K}$, van der Velde $\mathrm{K}$, Joosten $\mathrm{P}$, et al. Association between nutritional status and subjective health status in chronically ill children attending special schools. Qual Life Res 2016; 25:969-977.

53. Rub GML, Poraz I, Hartman $\mathrm{C}$, et al. Validation of a nutritional screening tool for ambulatory use in pediatrics. J Pediatr Gastroenterol Nutr 2016; 62:771-775.

54. Carey A, McCarthy $\mathrm{H}$, Thompson A, et al. A pilot study evaluating the use of the $\operatorname{STAMP}((\mathrm{c}))$ nutrition screening tool in hospitalised infants. Clin Nutr ESPEN 2015; 10:e192.

55. Johnson MJ, Pearson F, Emm A, et al. Developing a new screening tool for nutritional risk in neonatal intensive care. Acta Paediatr 2015; 104:e90-e93. 